\title{
7 Vergleichende Diskussion und Perspektiven der Forschung
}

Ziel der vorliegenden Untersuchung war es, dem Ursprung des Amokphänomens auf den Grund zu gehen, um einerseits einen Beitrag zum Verständnis des klassischen Amoklaufs zu leisten und andererseits zu helfen, School Shootings besser zu verstehen. Im Zentrum stand die Frage, ob School Shooting als schulgebundene Unterform des Amoklaufs betrachtet werden kann oder ob es sich um ein eigenständiges Phänomen handelt.

Im folgenden Kapitel werden die Untersuchungsergebnisse diskutiert und in den Kontext des gegenwärtigen wissenschaftlichen Forschungsstandes eingeordnet. Die Struktur des Kapitels orientiert sich an der Reihenfolge der untersuchten Gegenstandsbereiche. Zunächst werden die zentralen Befunde zu Amoklauf behandelt. Anschließend werden die Ergebnisse mit den Befunden zu School Shooting verglichen und die Frage erörtert, ob die Begriffe „Schulamoklauf" und "School Shooting“ synonym verwendet werden können. Ein abschließendes Fazit stellt die wichtigsten Erkenntnisse sowie die sich daraus ergebenden praktischen Konsequenzen noch einmal zusammenfassend dar.

\subsection{Individueller und kollektiver Amoklauf}

Auf der Basis des untersuchten Quellenmaterials lassen sich eine individuelle und eine kollektive Form des Amoklaufs unterscheiden. Beide Amokformen weisen zahlreiche Gemeinsamkeiten auf, unter anderem den gelegentlich die Handlung einleitenden und begleitenden Kampf- bzw. Warnruf „Amok“. Der aus der austronesischen Sprachfamilie entlehnte Begriff leitet sich von dem malaiischen Wort „mĕngamok“ ab, das ins Deutsche übersetzt „wütend angreifen“ bedeutet.

Im Südwesten Indiens und in Teilen des südostasiatischen Raums war kollektiver Amoklauf eine bis in das 17. Jahrhundert eingesetzte, mit dem nordgermanischen Berserkergang vergleichbare ritualisierte Kriegshandlung hoch angesehener Soldaten, die bereit waren für ihre Herrscher zu sterben und dabei so viele Feinde wie möglich mit in den Tod zu reißen. Die kollektive Form des Amoklaufs hat sich vermutlich von der Südwestküste Indiens ausgehend auf malaiische und javanische Gebiete Südostasiens ausgebreitet, weshalb gemeinschaftlicher Amoklauf als Kriegstaktik möglicherweise indischen Ursprungs ist. Dieser Befund deckt sich mit der Untersuchung des Schweizer Historikers Jörg Fisch, der am Beispiel der indischen Witwenverbrennung frühe, bis ins 6. Jahrhundert n. Chr. zurückreichende, indische (hinduistische) Einflüsse auf die Inseln Java und Bali nachweisen konnte. ${ }^{1}$ 
Bemerkenswert ist die Konzentration des Quellenmaterials zu kollektiv-militärtaktischen Amokläufen auf portugiesische und italienische Berichte aus dem 16. und 17. Jahrhundert. Dieser Befund verwundert vor dem Hintergrund des aggressiven und durchaus auch militärgestützten Vordringens der Portugiesen nach Asien jedoch nicht. Um die Handels- und Missionsinteressen der portugiesischen Krone durchzusetzen und den Seehandel in Asien zu kontrollieren, eroberten sie im 16. Jahrhundert entlang der malaiischen Küsten existierende Handelsplätze von Einheimischen, Chinesen und Arabern und errangen eine klare Vormachtstellung am Meer und die Kontrolle über die Pfeffer- und Gewürztransporte nach Europa, bis die Niederlande ihnen im 17. Jahrhundert diese Stellung streitig machten. ${ }^{2}$

Ebenso plausibel wie die Begegnung der Portugiesen mit der kriegerischen Form des Amoklaufs erscheint das Verschwinden des kollektiv-militärtaktischen Amokphänomens im Lauf des 17. Jahrhunderts. Angesichts der waffentechnologischen Fortschritte insbesondere im Bereich der Artillerie hat sich kollektiver Amok als Nahkampftaktik vermutlich zunehmend als wirkungslos erwiesen, weshalb Beschreibungen kollektiv-militärtaktischer Amokläufe im 19. Jahrhundert nur noch als Teil der Geschichtsschreibung aufzufinden sind.

Für die Authentizität der portugiesischen und italienischen Berichte und die Existenz des kollektiven Amokphänomens als militärtaktisches Kampfverhalten im malaiischen Raum spricht neben den bereits genannten Faktoren auch die Beschreibung kriegerischer Amokläufe in malaiischen Quellen wie der zwischen 1610 und 1620 verfassten Chronik „Sějarah Mĕlayu“ und dem zwischen 1650 und 1750 verfassten Heldenepos „Hikayat Hang Tuah“. Darin werden Amokkriegern heroische Eigenschaften wie Mut und Tapferkeit zugeschrieben. Dieser Befund deckt sich mit den Arbeiten anderer Autoren. In seiner „Geschichte der klassischen malaiischen Literatur“ zeigt Liaw Yock Fang am Beispiel der drei Heldenepen „Hikayat Sama’un“, „Hikayat Panji Kuda Semirang“ und „Hikayat Pandawa“, wie kriegerischer Amoklauf in der klassischen malaiischen bzw. javanischen Literatur thematisiert wird. ${ }^{3}$

Mit der Aufnahme des kriegerischen Amoklaufs in die klassische malaiische Literatur zeichnet sich eine weitere Gemeinsamkeit mit dem eingangs erwähnten Berserkergang nordgermanischer Krieger ab, der u. a. in der Schlacht am Hafrsfjord um 872 n. Chr. zum Einsatz gekommen sein soll und ebenfalls Inhalt zahlreicher Sagen und Mythen ist. ${ }^{4}$ Die Gegenüberstellung dieser Befunde lässt auf eine wechselseitige Inspiration zwischen malaiischem Heldenepos und realem Kriegsgeschehen schließen. Diese Schlussfolgerung wird durch Hans Overbeck am Beispiel des Heldenepos „Hikayat Amir Hamzah“ unterstützt, das bei den Malaien „sehr hoch geschätzt und vor einem Kampfe gelesen [wird], um aus den Schilderungen der Heldentaten Mut zu eigenen zu schöpfen" ". 
Kollektiver Amok war folglich einerseits ein real existierendes, ritualisiertes militärtaktisches Kampfverhalten und zugleich Schlachtruf besonders tapferer Ausnahmekrieger, die bereit waren, für Ruhm und Sold für ihren Herrscher zu sterben und möglichst viele Feinde mit in den Tod zu reißen, und andererseits Inhalt mythisch überhöhter Heldengeschichten, die der männlichen Bevölkerung als Vorbild für eigene „Heldentaten“ dienen sollten. Da der Konsonant am Ende des Wortes Amok im Malaiischen als finaler Stimmritzenverschluss (,glottal stop“) artikuliert wird, prädestinierten aus sprachlicher Sicht sowohl Kürze als auch Vokalität das malaiische Wort für seine Verwendung als Schlachtruf zur Einschüchterung des Feindes und gemeinsames Signal zum bedingungslosen Kampf.

Im kollektiven Bewusstsein war der Amokruf demnach lange Zeit mit den Heldentaten besonders furchtloser Krieger assoziiert. Individuelle Amokläufer konnten in den heldenhaften Darstellungen der Amokkrieger einerseits mythische Vorbilder für ihre eigenen Taten finden und durch deren Nachahmung andererseits versuchen, sich in die gesellschaftlich hoch angesehene Tradition kollektiver Amokläufer einzuschreiben. Die Häufigkeit, mit der Situationen von Gesichtsverlust bzw. Angriffe auf das Selbstkonzept als Auslöser für individuellen Amok berichtet werden, stützt diese Interpretation. Es spricht viel dafür, dass individuelle Amokläufer über die Identifikation mit den heroischen Vorbildern versucht haben könnten, ihre verletzte Ehre und Selbstachtung postum wiederherzustellen.

Die untersuchten Quellen belegen, dass individueller Amoklauf bis ins frühe 19. Jahrhundert ein auf einzelnen Inseln des Malaiischen Archipels und Teilen der Malaiischen Halbinsel verbreitetes und gefürchtetes Phänomen gewesen ist. Auf Java verfügten Polizeistationen über eigens für den Einsatz gegen individuelle Amokläufer vorgesehene Forken, die dazu dienten, Amokläufer auf Abstand zu halten und ihren rasenden Lauf ohne Gefahr für das eigene Leben zu stoppen. Aus den untersuchten Berichten geht hervor, dass es sich bei individuellen Amokläufern größtenteils um malaiische Männer zwischen dem zwanzigsten und vierzigsten Lebensjahr handelte. Fast immer wurden Klingenwaffen verwendet, häufig ein Kris oder Parang. Begünstigt wurde das gehäufte Auftreten des individuellen Amokphänomens durch die ständige Verfügbarkeit von Klingenwaffen. Überlieferungen aus dem frühen 16. Jahrhundert zufolge verließ auf Java kein Mann zwischen dem zwölften und dem achtzigsten Lebensjahr das Haus, ohne einen Kris an seinem Gürtel zu tragen. Eine mit einem Kris oder einem Parang ausgeführte Tat bedurfte folglich keiner langen Planung.

Der individuellen Variante des malaiischen Amoklaufs ging häufig ein depressives Vorstadium voraus. Ein „Pĕng-âmok“ genannter Einzeltäter ergriff plötzlich und ohne von außen beobachtbare unmittelbare Provokation seinen Kris oder Parang und stach wahl- und unterschiedslos jeden nieder, der in seinen Weg kam, bis er selbst getötet oder überwältigt wurde. Überlebende Amokläufer berichteten, dass ihnen plötzlich „mata gelap“ (wörtlich: schwarz vor Augen) geworden wäre. Für die 
Dauer des Amoklaufs wurde in der Regel eine komplette oder partielle Amnesie angegeben. Als häufige Auslöser für individuellen Amok wurden die drohende Versklavung zahlungsunfähiger Schuldner, persönliche Verluste, öffentliche Beleidigung oder Zurückweisung sowie Gesichtsverlust angeführt. Mit der Zunahme an Lebendfestnahmen unter der britischen Kolonialregierung und dem Aufbau von psychiatrischen Anstalten nach westlichem Vorbild wurden zunehmend auch infektiöse Ursachen wie Malaria oder Neurosyphilis sowie akute schizophrene Psychosen und Epilepsie als Auslöser für individuelle Amokläufe diskutiert. Medizinische Fortschritte in der Behandlung infektiöser Ursachen wie Malaria oder Neurosyphilis einschließlich der Therapie von akuten schizophrenen Psychosen und Epilepsien könnten daher den Rückgang der Amokinzidenz im malaiischen Raum in Teilen erklären.

Die Tatsache, dass individuelle Amokläufer ihren eigenen Tod billigend in Kauf nahmen bzw. diesen möglicherweise sogar in Sinne eines "suicide by cop“ bewusst intendierten, legt darüber hinaus einen Zusammenhang zwischen Fremd- und Selbsttötungsintention nahe. Diese Schlussfolgerung kann durch zeitgenössische Forschungsbefunde gestützt werden, die ebenfalls einen Zusammenhang zwischen Amok und Suizid nahelegen. Eine nichtrandomisierte Vergleichsstudie von Amokläufern aus Südostasien und US-amerikanischen Tätern fand eine durchschnittliche Suizidrate unter Amoktätern von mehr als fünfzig Prozent. ${ }^{6}$ Auch die Untersuchung von Haenel kommt zu dem Schluss, dass es sich bei „eigentliche[m] Amok“ um „indirekten Suizid“ handelt, bei dem der Täter einen Mord „benötigt“, um die Hemmschwelle zur Selbsttötung zu durchbrechen bzw. um getötet zu werden. ${ }^{7}$

Die regionale Konzentration des individuellen Amokphänomens auf den malaiischen Raum und die Nähe zwischen Fremd- und Selbsttötungsintention lassen den Schluss zu, dass es sich in vielen Fällen von individuellem Amok um eine sozial standardisierte und ritualisierte Form der Selbsttötung im Sinne eines „Amok-Suizids“ handelte. Das vorherrschende Suizidverbot erlaubte es den Mitgliedern der Gemeinschaft nicht, in (scheinbar) ausweglosen Situationen wie einer drohenden Sklaverei oder dem Verlust der Familie ihrem Leben durch eigene Hand ein Ende zu setzen. Zugleich lieferte der malaiische Heldenkult tollkühner Amokkrieger eine Handlungsschablone, die es ermöglichte, in sozial standardisierter und kulturell anerkannter Weise aus dem Leben zu scheiden: „[E]s ist ganz so, als sagte die Gruppe dem Individuum: , Tu es nicht, aber wenn du es tust, dann muß es so und so gemacht wer-

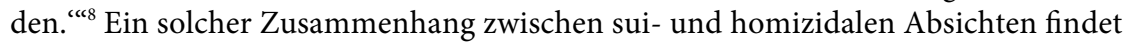
sich gemäß Karl Menninger häufig im Rahmen suizidaler Handlungen. Der Psychiater unterscheidet drei Suizidmotive: 1. Den Wunsch zu töten, 2. den Wunsch, getötet zu werden und 3. den Wunsch zu sterben. ${ }^{9}$

In der Zusammenschau der Befunde erscheint es somit höchst wahrscheinlich, dass die regionale und kulturelle Konzentration des individuellen Amokphänomens auf 
die Gebiete des heutigen Indonesien und Malaysia maßgeblich durch die Kombination aus Suizidverbot, Heldenkult und ständiger Verfügbarkeit von Klingenwaffen begünstigt wurde. Mit der zunehmenden Pathologisierung individueller Amoktaten und der steigenden Zahl an Lebendfestnahmen im Zuge der Kolonialisierung sank die Amokprävalenz entsprechend, da es für Amokläufer zunehmend unsicherer wurde, durch ihre Handlungen zum Tode zu gelangen.

\subsection{School Shooting und Amoklauf}

Während individueller Amoklauf vor rund 600 Jahren durch europäische Fernreisende erstmals in den Gebieten des heutigen Indonesien und Malaysia beschrieben wurde, sind von School Shooting vor allem öffentliche Bildungseinrichtungen in Staaten der westlichen Welt betroffen. Von den 160 bislang für den Zeitraum 1900 bis 2013 dokumentierten School Shootings wurden 72 Prozent in Nordamerika verübt. Von den übrigen 28 Prozent wurde die Hälfte in Europa ausgeführt. ${ }^{10}$ Auf den ersten Blick besitzen Amoklauf und School Shooting folglich sowohl Unterschiede im zeitlichen als auch im räumlichen Auftreten des Phänomens. Im Zentrum der vorliegenden Untersuchung stand die Frage, ob Gemeinsamkeiten oder Unterschiede hinsichtlich bestimmter Tätermerkmale sowie in Bezug auf Entstehung und Ausführung der Taten überwiegen und ob School Shooting als „schulgebundene Unterform des Amoklaufs" ${ }^{\text {"11 }}$ betrachtet werden kann. Sowohl in deutschsprachigen Medienberichten als auch in Fachpublikationen werden die Begriffe School Shooting und Schulamoklauf häufig synonym verwendet. ${ }^{12}$ So werden „Amoktaten“, die an Schulen begangen werden, auch als "Schulamok“ oder "Schulamoklauf" bezeichnet.

Die detaillierte Untersuchung zweier klassischer Fälle von School Shooting in den Abschnitten 6.1.1 und 6.1.2 der vorliegenden Arbeit zeigt auf der Grundlage zahlreicher Quellenbelege, dass School Shooter nicht aus einem plötzlichen Impuls heraus handeln. Die Tatbereitschaft entwickelt sich selten spontan, sondern steht am Ende eines langen Radikalisierungsprozesses. School Shooter planen ihre Taten langfristig und gezielt. Es handelt sich in der Regel um Jugendliche oder junge Erwachsene männlichen Geschlechts mit einer ausgeprägten Affinität zu Schusswaffen und dem Konsum gewalthaltiger Medien. Sie inszenieren sich bevorzugt als Opfer und einsame Rächer und verfassen mitunter Selbstzeugnisse, in denen sie ihr Vorgehen rechtfertigen.

Die Täter stammen häufig aus gut situierten, äußerlich intakten Mittelschichtsfamilien. Die Wahl der eigenen (aktuellen oder ehemaligen) Schule als Tatort erfolgt bewusst. School Shooter suchen sich nicht irgendeine Bildungseinrichtung für ihre Tat aus, sondern diejenige, zu der sie eine persönliche Beziehung besitzen. Bei der Frage, ob und wann die Tatpläne umgesetzt werden, spielen bestimmte Triggerfaktoren wie 
die mediale Berichterstattung über eine andere Tat oder Jahrestage von spektakulären Taten eine Rolle. Häufig wird das Tatgeschehen vorher im Rahmen von konkreten Tötungsfantasien antizipiert, wodurch ein (kompensatorisches) Gefühl von Überlegenheit, Kontrolle und Macht entsteht. In diese Phase fallen auch Äußerungen über geplante Tötungsabsichten, häufig in Form indirekter Andeutungen.

Geleitet werden die Täter von einem Motivbündel aus Hass, narzisstischer Wut als Reaktion auf erfahrene oder empfundene Kränkungen, depressiven Affekten, Todeswünschen sowie dem Wunsch nach Rache. Häufig bestehen Suizidabsichten. Das School Shooting wird instrumentalisiert, um größtmögliche mediale Aufmerksamkeit zu erzielen. Anders als bei Massentötungen durch erwachsene Täter (siehe Abschnitt 6.2.2) spielen psychotisch bedingte Motive in der Regel keine Rolle. Stattdessen orientieren sich School Shooter klassischerweise an medialen und realen Vorbildern, über die sie im Vorfeld im Internet recherchieren und denen sie in ihrem Streben nach medialer Bekanntheit und Anerkennung nacheifern. Dieser Befund steht im Einklang mit Untersuchungsergebnissen aus dem Bereich der sozial-kognitiven Lerntheorie (siehe Abschnitt 4.3.3). Neuere Untersuchungen zum Einfluss der medialen Berichterstattung auf jugendliche Nachahmungstäter bestätigen dieses als „Copycat-“ oder "Columbine-Effekt" bezeichnete Nachahmungsphänomen. ${ }^{13}$

Die Befunde aus den beiden für die vorliegende Arbeit untersuchten School Shootings decken sich mit aktuellen Forschungsbefunden, denen eine höhere Fallzahl zugrunde liegt. ${ }^{14}$ Die vergleichende Untersuchung zeigt, dass sich School Shooter und Amokläufer hinsichtlich bestimmter Merkmale wie Alter, Bewusstseinszustand sowie Einsichts- und Steuerungsfähigkeit zum Tatzeitpunkt deutlich unterscheiden. School Shooter sind im Durchschnitt etwa zehn bis zwanzig Jahre jünger als Amokläufer. Sie handeln mehrheitlich bei klarem Bewusstsein. Im Gegensatz zu klassischen Amokläufern gehen sie sehr kontrolliert und zielgerichtet vor und das über einen längeren Zeitraum hinweg, der von Minuten bis Stunden reichen kann, wie aus Videoaufnahmen der Taten an Schulen eindeutig hervorgeht. ${ }^{15}$ Eine tatbezogene Amnesie besteht in der Regel nicht. Es gibt auch keine Hinweise auf ein kurz vor der Tat eintretendes Schwarzwerden vor Augen („mata gelap") oder auf eine blinde, d.h. ungerichtete Wut und Raserei. School Shooter rasten nicht plötzlich aus. Stattdessen handeln die Täter ruhig und überlegt und töten in abgekühlter Gemütslage („,old anger ${ }^{\text {“16) }}$. Anders als Amokläufer empfinden School Shooter auch keine Reue für ihre Tat.

Während einem School Shooting in der Regel über einen längeren Zeitraum gewachsene Planungen vorausgehen, ereignen sich Amokläufe klassischerweise ohne lange und gezielte Planung. Der Amoklauf ähnelt phänotypisch vielmehr einem katatonen Erregungssturm, der durch eine ungerichtete motorische Erregungsphase mit schwerster blindwütiger Aggression gegenüber zufällig anwesenden Menschen cha- 
rakterisiert ist. Nicht selten sind Bekannte und Verwandte des Amokläufers die ersten Opfer, bevor der Amoklauf auf Unbeteiligte ausgeweitet wird. Der Angriff erfolgt häufig im Rahmen eines Dämmerzustands oder einer Verwirrtheit, wie sie beispielsweise bei akuter Belastung, schweren depressiven Zuständen oder in Folge einer Psychose auftreten können. Es wird regelhaft ein kurz vor der Tat eintretendes Schwarzwerden vor Augen („mata gelap“) beschrieben. Während der Tat ist das Bewusstsein eingeengt und getrübt. Für die Dauer des Dämmerzustands besteht häufig eine Amnesie.

Dieser Gegensatz zwischen heftiger motorischer Erregungsphase und abgekühlter Affektlage mit erhaltener Selbstkontrolle bildet sich am besten in der Unterscheidung zwischen impulsiver und instrumenteller Aggression ab. Während klassischer Amok dem Bereich der impulsiven, ungerichteten Aggression zugeordnet werden kann, handelt es sich bei School Shooting um eine Form instrumenteller, zielgerichteter Aggression.

Dieser Untersuchungsbefund deckt sich mit aktuellen Forschungsbefunden, wonach sich School Shootings junger Täter von Massentötungen durch erwachsene Täter deutlich unterscheiden. ${ }^{17}$ Die im Abschnitt 6.2.2 untersuchte Massentötung vom 20. Juni 1913 an einer Bremer Schule durch einen erwachsenen Täter weist dementsprechend deutliche Unterschiede zu klassischen School Shootings durch junge Täter auf. Der Täter stand in keiner direkten persönlichen Beziehung zu der als Tatort gewählten Bildungseinrichtung. Er handelte im Rahmen eines paranoiden Wahns und war zum Tatzeitpunkt psychotisch. Es ging dem Täter nicht darum, ein öffentliches Medienecho zu erzeugen oder seinem Leben ein Ende zu setzen, sondern er fühlte sich verfolgt und bedroht. Aufgrund des fehlenden impulsiven oder raptusartigen Beginns und der Zielgerichtetheit bezüglich des Tatorts erfüllt der Bremer Massenmord allerdings auch nicht die Kriterien eines klassischen Amoklaufs. Die Tat unterscheidet sich zudem deutlich von der im Abschnitt 6.2.1 untersuchten Bluttat am Saarbrücker Gymnasium vom 25. Mai 1871 durch einen Schüler, die zahlreiche Gemeinsamkeiten mit klassischen School Shootings junger Täter aufweist.

\subsection{Notwendigkeit eines Paradigmenwechsels}

Die vergleichende Untersuchung von Malaiischem Mĕngamok und School Shooting hat gezeigt, dass die Unterschiede hinsichtlich bestimmter Tätermerkmale sowie in Bezug auf Entstehung und Ausführung der Taten deutlich überwiegen. Entgegen einem weit verbreiteten Mythos handelt es sich bei School Shootings nicht um spontane Affekttaten. Das klassische Konzept des Amoklaufs als impulsive Spontantat greift für School Shootings folglich nicht. Aus diesen Gründen sollten sogenannte „Amokläufe“ an Schulen nicht als Amok bezeichnet, sondern in Abgrenzung zu diesem Begriff anders klassifiziert werden. 
Die lange und z. T. minutiöse Tatvorbereitung unterscheidet School Shootings von Amokläufen und rückt diese extreme Form zielgerichteter Gewalt an Schulen in die Nähe von terroristischen Anschlägen von Einzeltätern. Dieser Befund deckt sich mit aktuellen Forschungsbefunden. In ihrer Vergleichsstudie zeigten Lankford und Hakim, dass sich US-amerikanische Täter sogenannter „Rampage Shootings“ (zu denen School Shootings zählen) und Selbstsprengungsattentäter des Nahen Ostens ähneln. ${ }^{18}$ Auch in der deutschsprachigen Forschung mehren sich Hinweise, wonach School Shootings in ihrer Entwicklung und Durchführung terroristischen Anschlägen sogenannter "lone actors“ ähneln. ${ }^{19}$ Robertz und Kahr wiesen zudem darauf hin, dass School Shooter genau wie terroristische Einzeltäter die mediale Wirkung ihrer Taten bewusst einkalkulieren. ${ }^{20}$

Angesichts der Ergebnisse aus der vorliegenden Untersuchung erscheint es passender, für schwere zielgerichtete Gewalttaten aktueller oder ehemaliger Schüler mit persönlichem Bezug zu einer bewusst als Tatort ausgewählten Bildungseinrichtung den Begriff „Schulanschlag“ zu verwenden, anstatt von „Schulamokläufen“ zu sprechen. Unter einem „Anschlag“ wird sowohl ein „gewalttätiger, auf Vernichtung, Zerstörung zielender Angriff“ verstanden als auch eine „Bekanntmachung, die am Schwarzen Brett, an einer Mauer, Litfaßsäule o. Ä. angeschlagen ist ${ }^{\text {“21 }}$. Der doppeldeutige Begriff "Schulanschlag" hat folglich den Vorteil, zugleich das Motiv der öffentlichen Aufmerksamkeit und den gewalttätigen Angriff auf Lehrer und Schüler einer Schule zu bezeichnen. Darüber hinaus klingt die Bezeichnung "Schulanschlag“ weder verharmlosend noch schränkt sie das Phänomen auf den Gebrauch von Schusswaffen ein. Alternativ kann auch der Begriff „Schulmassaker“ in Erwägung gezogen werden. Das Wort „Massaker“ („Hinmorden einer großen Anzahl [unschuldiger, wehrloser], Menschen; Blutbad“'22) beinhaltet jedoch nicht den doppeldeutigen Aspekt des „Anschlags", der das Phänomen so treffend beschreibt.

Andere Begriffe wie „Angriff“ („Eröffnung eines Kampfes“) oder „Attacke“ („scharfe Kritik, Feldzug gegen etwas“) sind zu allgemein, der Begriff „Attentat“ („politisch od. ideologisch motivierter [Mord] anschlag auf eine im öffentlichen Leben stehende Persönlichkeit") zu spezifisch, um den Begriff "School Shooting“ besser zu beschreiben. ${ }^{23}$ Von einem "Schulanschlag" anstatt von einem „Schulamoklauf" zu sprechen, hieße allerdings anzuerkennen, dass es sich nicht um ein Phänomen fernen Ursprungs handelt, sondern um ein Phänomen, das seinen Ursprung in unserer westlich geprägten Gesellschaft hat und eng mit dieser assoziiert ist. Dies hieße, sich einzugestehen, dass unsere Kultur einen maßgeblichen Anteil an der Entwicklung und Aufrechterhaltung dieses Phänomens hat, eröffnet aber auch völlig neue Perspektiven der Prävention dieser schweren Gewalttaten. 


\subsection{Fazit und Ausblick}

Die Ergebnisse der vorliegenden Untersuchung zu Amoklauf und School Shooting geben Anlass, sowohl die Nomenklatur als auch die Klassifikation sogenannter "Schulamokläufe" zu revidieren. Sie werfen darüber hinaus ein neues Licht auf die School-Shooting-Forschung, die noch vor wenigen Jahren von einem ersten Auftreten des Phänomens auf deutschem Boden in den 1990er-Jahren ausging.

Im Folgenden sollen die wichtigsten Ergebnisse sowie die sich daraus ergebenden praktischen Konsequenzen noch einmal zusammenfassend dargestellt werden:

- Auf der Basis des recherchierten Quellenmaterials lassen sich eine individuelle und eine kollektive Form des Amoklaufs unterscheiden. Beide Amokformen weisen zahlreiche Gemeinsamkeiten auf, unter anderem den gelegentlich die Handlung einleitenden und begleitenden Kampf- bzw. Warnruf „Amok“.

- Der Begriff leitet sich von dem malaiischen Wort „mĕngamok“ ab, das ins Deutsche übersetzt „wütend angreifen“ bedeutet. Da der Konsonant am Wortende als finaler Stimmritzenverschluss artikuliert wird, prädestinierten sowohl Kürze als auch Vokalität das malaiische Wort für seine Verwendung als Schlachtruf zur Einschüchterung des Feindes und gemeinsames Signal zum bedingungslosen Kampf.

- Im Südwesten Indiens und in Teilen des südostasiatischen Raums war kollektiver Amoklauf eine mit dem nordgermanischen Berserkergang vergleichbare ritualisierte Kriegshandlung hoch angesehener Soldaten, die bereit waren für ihre Herrscher zu sterben und dabei so viele Feinde wie möglich mit in den Tod zu reißen.

- Die erste hier recherchierte Schilderung von kollektivem Amoklauf befindet sich in in den „Lendas da Índia“ Gaspar Correas. Der portugiesische Schreiber berichtet darin den gemeinschaftlichen Amoklauf mehrerer indischer ,amoucos" anlässlich des Krieges zwischen den Königen von Cochin und Kalikut im Jahre 1503 .

- Kriegerische Amokläufer standen in hohem Ansehen. Ihr Verhalten war mit positiven Eigenschaften wie Stärke, Furchtlosigkeit und Loyalität assoziiert. In bestimmten Situationen galt ihr Überleben jedoch als Schande. Zur Wiederherstellung der Ehre wurde von ihnen erwartet, dass sie in das Feindesland zogen und dort wahllos alles und jeden niederstießen, bis sie selbst getötet wurden. 
- Individuellen Amokläufern diente das kollektive Amokverhalten möglicherweise als Vorbild. Ein „Pěng-âmok“ genannter Einzeltäter ergriff plötzlich seinen Dolch und stach ungezielt jeden nieder, der in seinen Weg kam, bis er selbst getötet oder überwältigt wurde.

- Oftmals befanden sich Freunde oder Familienangehörige des Amokläufers unter den ersten Opfern. Der Amok wurde anschließend auf Unbeteiligte erweitert, möglicherweise mit dem Ziel, dabei selbst getötet zu werden. Der Amokläufer stürmte dann aus dem Haus und tötete oder verletzte wahllos jeden, der sich ihm in den Weg stellte.

- Begünstigt wurde die regionale und kulturelle Konzentration des individuellen Amokphänomens auf die Gebiete des heutigen Indonesien und Malaysia durch die Kombination aus Suizidverbot, Heldenkult und ständiger Verfügbarkeit von Klingenwaffen.

- Die erste hier recherchierte Schilderung von individuellem Amoklauf befindet sich in einem Bericht des venezianischen Kaufmanns Niccolò di Conti, der etwa im Jahr 1415 von Venedig aus über die arabische Welt nach Indien und bis nach Sumatra und Java gereist war.

- Der malaiische Heldenkult tollkühner Amokkrieger lieferte eine Handlungsschablone, die es ermöglichte, in scheinbar ausweglosen Situationen in sozial standardisierter und kulturell anerkannter Weise aus dem Leben zu scheiden. Eine mit einem Kris oder einem Parang ausgeführte Tat bedurfte zudem keiner langen Planung.

- Individuellem Amoklauf ging häufig eine Periode depressiven Brütens voraus, die als „sakit hati“ bezeichnet wurde. Überlebende Einzeltäter berichteten, vor dem Amoklauf „mata gelap“ gewesen zu sein, was so viel bedeutet wie, dass ihnen schwarz vor Augen geworden sei und sie nicht Herr ihrer Sinne gewesen seien. Für die Amokhandlungen selbst wurde in den meisten Fällen totale oder zumindest partielle Amnesie angegeben.

- Entgegen einem weit verbreiteten Mythos handelt es sich bei School Shootings nicht um spontane, affektgesteuerte Impulstaten. School Shootings zeichnen sich durch eine intensive Planungsphase und ein kontrolliertes, zielgerichtetes Vorgehen aus. Anders als bei Massentötungen durch erwachsene Täter spielen psychotisch bedingte Motive zum Tatzeitpunkt in der Regel keine Rolle.

- Die Wahl der eigenen (aktuellen oder ehemaligen) Schule als Tatort erfolgt bewusst. Geleitet werden die meist jugendlichen Täter von einem Motivbündel aus Hass, narzisstischer Wut als Reaktion auf erfahrene oder empfundene 
Kränkungen, depressiven Affekten, Todeswünschen sowie dem Wunsch nach Rache.

- Die untersuchte Massentötung vom 20. Juni 1913 an einer Bremer Schule durch einen erwachsenen Täter weist deutliche Unterschiede zu klassischen School Shootings durch junge Täter auf. Aufgrund des fehlenden impulsiven oder raptusartigen Beginns und der Zielgerichtetheit bezüglich des Tatorts erfüllt der Bremer Massenmord allerdings auch nicht die Kriterien eines klassischen Amoklaufs. Der Täter handelte im Rahmen eines paranoiden Wahns und war zum Tatzeitpunkt psychotisch.

- Die Einbeziehung eines School Shootings aus dem Jahr 1871 in die Untersuchung und der Vergleich mit jüngeren School Shootings hat es ermöglicht, Veränderungen bzw. Entwicklungen des Phänomens zu identifizieren. $\mathrm{Zu}$ nennen ist hier vor allem der Einfluss der mittlerweile weltweiten medialen Berichterstattung auf Nachahmungstäter („Columbine-Effekt“).

- School Shooter orientieren ihre Tatausführung an medialen und realen Vorbildern, über die sie im Vorfeld im Internet recherchieren. Das Streben nach Bekanntheit und größtmöglicher medialer Aufmerksamkeit war im 19. Jahrhundert hingegen nicht maßgeblich. Stattdessen handelte der Saarbrücker Gymnasiast unter den Eindrücken des Deutsch-Französischen Krieges und fand möglicherweise in den noch vereinzelt üblichen Pistolenduellen unter „Ehrenmännern" ein Skript für seine Tat.

Die vorliegende Untersuchung widerlegt die weit verbreitete Annahme, dass es sich bei School Shooting um eine schulgebundene Unterform des Amoklaufs handelt. Vergleiche zeigen, dass die Unterschiede hinsichtlich bestimmter Tätermerkmale sowie in Bezug auf Entstehung und Ausführung der Taten deutlich überwiegen. Die lange und zum Teil minutiöse Tatvorbereitung unterscheidet School Shootings vom klassischen Amoklauf und rückt diese Taten in die Nähe von zielgerichteten terroristischen Anschlägen von Einzeltätern. Angesichts der Ergebnisse aus der vorliegenden Untersuchung sollten School Shootings folgerichtig nicht als "Schulamokläufe“, sondern als „Schulanschläge“ bezeichnet werden.

Inwiefern School Shooter tatsächlich Gemeinsamkeiten mit anderen Tätergruppen wie terroristischen Einzeltätern („lone actors") haben, müssen weitere Studien zeigen. Auch vergleichende Untersuchungen mit Mehrfachtötungen am Arbeitsplatz („workplace shootings") oder an Universitäten („campus shootings") sind wünschenswert, um etwaige Ähnlichkeiten oder aber Unterschiede zu School Shootings herauszuarbeiten. Die vergleichende Untersuchung von School Shootern und diesen Tätergruppen könnte ein tieferes Verständnis dieser schwerwiegenden Gewalttaten 
ermöglichen und bei der Entwicklung präventiver Konzepte, die der Ausführung solcher Taten entgegenwirken, hilfreich sein.

Für die vertiefende Untersuchung des malaiischen Amokphänomens wäre es zudem wünschenswert, weitere malaiische Quellen zu identifizieren und für zukünftige Studien im Bereich der Amokforschung miteinzubeziehen. Dabei wäre es auch interessant, das Forschungsmaterial auf andere außereuropäische Quellen auszuweiten und beispielsweise Reiseberichte chinesischer buddhistischer Pilgermönche oder arabischer Südostasienreisender auf Amokschilderungen zu untersuchen. Von besonderem Interesse erscheint auch der internationale Vergleich von Amoktaten bzw. School Shootings, um kulturelle und landestypische Unterschiede oder Übereinstimmungen zu identifizieren und gesamtgesellschaftliche Einflussfaktoren wie z. B. die Verfügbarkeit von Waffen noch stärker herauszuarbeiten.

1 Vgl. Fisch, Jörg: Der indische Einfluß in Südostasien: Java und Bali. In: Ders.: Tödliche Rituale. Die indische Witwenverbrennung und andere Formen der Totenfolge. Frankfurt am Main [u.a.]: Campus 1998, S. 193-212.

2 Vgl. Feldbauer, Peter: Die Portugiesen in Asien 1498-1620. (Überarb. Neuauflage). Essen: Magnus 2005 [2003].

3 Vgl. Fang, Liaw Yock: A History of Classical Malay Literature. Aus dem Malaiischen übersetzt von Razif Bahari und Harry Aveling. Jakarta: Yayasan Pustaka Obor \& Institute of Southeast Asian Studies 2013, S. 97, S. 127 und S. 235.

4 Vgl. See, Klaus von: Exkurs zum Haraldskvæði: Berserker. In: Ders.: Edda, Saga, Skaldendichtung. Aufsätze zur skandinavischen Literatur des Mittelalters. (= Skandinavistische Arbeiten, Bd. 6). Heidelberg: Carl Winter 1981, S. 311-317.

5 Vgl. Overbeck, Hans: Einführung in die malaiische Literatur. In: Ders. (Übers.): Malaiische Weisheit und Geschichte. Einführung in die malaiische Literatur. Die Krone aller Fürsten. Die Chronik der Malaien. (= Insulinde, Bd. 1, Vom Goldenen Chersones). Jena: Diederichs 1927, S. 1-45, hier S. 24.

6 Vgl. Hempel, Anthony G.; Levine, Ruth E.; Meloy, J. Reid; Westermeyer, Joseph: A crosscultural review of sudden mass assault by a single individual in the oriental and occidental cultures. In: Journal of Forensic Sciences 45 (2000): 582-588, hier S. 583.

7 Vgl. Haenel, Thomas: Amok und Kollektivsuizid. Selbsttötung als Gruppenphänomen. München: Fink 2012, S. 93.

8 Devereux, Georges: Normal und anormal. In: Ders.: Normal und anormal. Aufsätze zur allgemeinen Ethnopsychiatrie. (= suhrkamp taschenbuch wissenschaft, Bd. 395). Aus dem Französischen übersetzt von Nils Thomas Lindquist. Frankfurt am Main: Suhrkamp 1982, S. 7-118, hier S. 52.

9 Vgl. Menninger, Karl A.: Selbstzerstörung. Psychoanalyse des Selbstmords. Aus dem Englischen übersetzt von Hilde Weller. (= suhrkamp taschenbuch wissenschaft, Bd. 249). Frankfurt am Main: Suhrkamp 1978 [1938], S. 39-97.

10 Vgl. Dumitriu, Camélia; Huțu, Carmen Aida: A Disaster Management Framework for Coping with Acts of Extreme Violence in School Settings. A Field Study. In: GRF Davos 
Planet@Risk 2 (2014): 101-116, hier S. 102.

11 Vgl. etwa Scheithauer, Herbert; Bondü, Rebecca: Amoklauf und School Shooting. Bedeutung, Hintergründe und Prävention. Göttingen: Vandenhoeck \& Ruprecht 2011, S. 24.

12 Vgl. etwa Sitzer, Peter; Böckler, Nils: Schulamok/School Shooting. In: Melzer, Wolfgang; Hermann, Dieter; Sandfuchs, Uwe; Schäfer, Mechthild; Schubarth, Wilfried; Daschner, Peter (Hgg.): Handbuch Aggression, Gewalt und Kriminalität bei Kindern und Jugendlichen. Bad Heilbrunn: Klinkhardt 2015, S. 275-278.

13 Vgl. Coleman, Loren: The Copycat Effect. How the Media and Popular Culture Trigger the Mayhem in Tomorrow's Headlines. New York [u. a.]: Paraview Pocket Books 2004; vgl. auch Roth, Daniel: Zündstoff für den „Columbine-Effekt“? Die Berichterstattung über School Shootings in deutschen Print- und Online-Medien. (= Medien \& Kommunikation, Bd. 30). Münster: LIT Verlag 2012.

14 Vgl. etwa Vossekuil, Bryan; Fein, Robert A.; Reddy, Marisa; Borum, Randy; Modzeleski, William: The Final Report and Findings of the Safe School Initiative. Implications for the Prevention of School Attacks in the United States. Washington D.C.: Unites States Secret Service and United States Department of Education 2004; vgl. auch Bannenberg, Britta: Schlussbericht Projekt TARGET. Teilprojekt Gießen. Kriminologische Analyse von Amoktaten - junge und erwachsene Täter von Amoktaten, Amokdrohungen im Verbundprojekt TARGET (Tat- und Fallanalysen hoch expressiver zielgerichteter Gewalt). Gießen: JustusLiebig-Universität, Fachbereich o1 Rechtswissenschaft, Professur für Kriminologie 2017. URL: http://www.uni-giessen.de/fbz/fbo1/professuren-forschung/professuren/bannenber g/mediathek/dateien/schlussbericht-target-giessen2017.pdf [Stand: 28. Juli 2018].

15 Vgl. etwa Video clip from the security cameras of the the Columbine shootings. URL: http:/ /www.acolumbinesite.com/security.php [Stand: 28. Juli 2018].

16 Meloy, J. Reid; Hempel, Anthony G.; Gray, B. Thomas; Mohandie, Kris; Shiva, Andrew; Richards, Thomas C.: A Comparative Analysis of North American Adolescent and Adult Mass Murderers. In: Behavioral Sciences and the Law 22 (2004): 291-309, hier S. 297.

17 Vgl. etwa Bannenberg, Britta; Bauer, Petra: Amoktaten. Phänomenologie und Hintergründe. In: Rechtsmedizin 3 (2017): 154-161.

18 Vgl. Lankford, Adam; Hakim, Nayab: From Columbine to Palestine. A comparative analysis of rampage shooters in the United States and volunteer suicide bombers in the Middle East. In: Aggression and Violent Behavior 16 (2011): 98-107.

19 Vgl. Leuschner, Vincenz; Böckler, Nils; Zick, Andreas; Scheithauer, Herbert: Attentate durch Einzeltäter. Zu Gemeinsamkeiten in der Tatentwicklung und der Tatsituation bei terroristischen Anschlägen und School Shootings. In: Böckler, Nils; Hoffmann, Jens (Hgg.): Radikalisierung und terroristische Gewalt. Perspektiven aus dem Fall- und Bedrohungsmanagement. Frankfurt am Main: Verlag für Polizeiwissenschaft 2017, S. 51-78.

20 Vgl. Robertz, Frank J.; Kahr, Robert (Hgg.): Die mediale Inszenierung von Amok und Terrorismus. Zur medienpsychologischen Wirkung des Journalismus bei exzessiver Gewalt. Wiesbaden: Springer 2016.

${ }^{21}$ Anschlag. In: Dudenredaktion (Hg.): Duden - Deutsches Universalwörterbuch. Das umfassende Bedeutungswörterbuch der deutschen Gegenwartssprache. (8., überarb. u. erw. Auflage). Berlin: Dudenverlag 2015, S. 159.

22 Massaker. In: Ebd., S. 1172.

23 Vgl. Angriff, Attacke und Attentat. In: Ebd., S. 148 und 191. 
Dieses Kapitel wird unter der Creative Commons Namensnennung 4.0 International Lizenz (http://creativecommons.org/licenses/by/4.0/deed.de) veröffentlicht.

(c) (1) 